\title{
Citogenética evolutiva en Leguminosas AMERICANAS
}

\author{
Lidia Poggio ${ }^{1,2}$, Shirley M. Espert ${ }^{1,2}$ \& Renée H. Fortunato ${ }^{2,3,4}$
}

\section{RESUMEN}

(Citogenética evolutiva en Leguminosas americanas) Se presentan las características cromosómicas descriptas hasta el momento en Leguminosas americanas. A través del análisis de estos datos en conjunto con los morfológicos y las filogenias moleculares se proponen hipótesis acerca de los cambios cromosómicos ocurridos durante el proceso de divergencia y especiación de la familia. Los estudios cromosómicos indican una gran variación intergenérica, inter e intraespecífica, además de una amplia diversificación en el tamaño del genoma entre géneros, especies y poblaciones. A partir del número básico ancestral $x=7$ se deduce que la especiación híbrida poliploide ha sido muy importante en la diversificación de la familia. Por procesos de disploidía creciente y decreciente, tanto a nivel diploide como poliploide se originarían números básicos secundarios y series poliploides modificadas. En la parafilética subfamilia Caesalpinioideae habría predominado el proceso de disploidia decreciente de $\mathrm{n}=14$ a $\mathrm{n}=11$. En la monofilética subfamilia Mimosoideae, ocurrió un evento principal de evolución del número cromosómico de 14 a 13. Por último en Papilionoideae, la subfamilia más derivada de Leguminosae, se observó reducción del número básico de 14 a 7, pasando por números gaméticos de 11 y 8 . Por otro lado, el origen recurrente de los poliploides y la ocurrencia de rearreglos intergenómicos, hibridación y poliploidía secundaria, son procesos que dificultan la agrupación natural de los taxones en algunos grupos de la familia Leguminosae.

Palabras clave: Leguminosae, citogenética, poliploidía, evolución cromosómica, hibridación, América.

\section{Abstract}

(Evolutionary cytogenetics in Americans Legumes) Chromosomic features described so far for Americans legumes are reviewed and analyzed, simultaneously with morphological and molecular characters. This is an invaluable opportunity to propose hypotheses about chromosome changes during the family divergence and speciation processes. The chromosome studies showed wide inter-generic, inter and intra-specific variability, as well as marked differences in genome sizes between genera, species and populations. By postulating an ancestral basic number of seven $(x=7)$ it is possible to deduce that polyploid hybrid speciation was very important in the diversification of the family as a whole. Due to increasing and decreasing diploidy processes, secondary basic numbers and modified polyploids series could have arised both at diploid and polyploid levels. On the other hand, recurrent origin of polyploids, occurrence of inter-genomic rearrangements, hybridization and secondary polyploidy are all processes that make it difficult to group the taxa within the Leguminosae family in a natural sequence.

Key words: Leguminosae, cytogenetics, poliploidía, chromosome evolution, hybridization, America.

\section{INTRODUCCIÓN}

La citogenética brinda valiosos aportes para la resolución de problemas taxonómicos, evolutivos y aplicados, contribuyendo al conocimiento del origen y evolución de distintos grupos. Dado que los cromosomas son guías de afinidades filogenéticas e indicadores de las clasificaciones sistemáticas es importante analizar, mediante técnicas de citogenética clásica y molecular (FISH, GISH), las características del cariotipo, el comportamiento meiótico en híbridos y poliploides, y la variación intra- e ínterespecífica en el tamaño del genoma. Asimismo los estudios citogenéticos permiten realizar valiosos aportes al conocimiento de los mecanismos de aislamiento reproductivo y modos de especiación en plantas.

La especiación híbrida es muy común en el reino vegetal, en especial la originada por poliploidía. Los estudios cromosómicos son

Artigo recebido em 09/2006. Aceito para publicação em 01/2008.

${ }^{1}$ Laboratorio de Citogenética y Evolución, Departamento de Ecología, Genética y Evolución, Facultad de Ciencias Exactas y Naturales, Universidad de Buenos Aires, Argentina.

${ }^{2}$ Consejo Nacional de Investigaciones Científicas y Técnicas (CONICET).

${ }^{3}$ Instituto de Recursos Biológicos, INTA, Castelar 1712, Provincia de Buenos Aires, Argentina.

${ }^{4}$ Autor para correspondencia: rfortunato@cnia.inta.gov.ar 
importantes para analizar la presencia de zonas híbridas con la finalidad de detectar la formación de nuevas subespecies por introgresión y conocer el impacto de la hibridación natural en la formación de complejos híbridos homoploides y/o poliploides (Grant 1985).

Los estudios citogenéticos permiten deducir el número básico, dato muy importante para proponer hipótesis filogenéticas en un grupo. Las principales características que aportan información a la evolución cromosómica son: posición del centrómero, número, tipo y posición de zonas organizadoras del nucleolo, tamaño absoluto y relativo de los cromosomas, cantidad y distribución de heterocromatina, composición del ADN repetido (por técnicas de citogenética molecular) y contenido de ADN total. Leitch et al. (1998) investigaron la evolución del tamaño del genoma en Angiospermas analizando en forma conjunta los valores del contenido de ADN existente y árboles filogenéticos robustos basados en caracteres moleculares y morfológicos. La superposición de ambos tipos de estudios permitieron concluir que las angiospermas ancestrales poseían genomas pequeños (menor o igual a 1,4 pg considerando $1 \mathrm{pg}=10-12 \mathrm{gr}=965 \mathrm{Mbp}$ ) siendo la adquisición de grandes genomas una condición derivada que habría surgido repetidamente en la evolución de distintas familias. La presencia del carácter ancestral (genomas pequeños) ha sido retenida en muchos grupos actuales. Soltis et al. (2003) confirman que los genomas mayores ocurren en clados que ocupan posiciones derivadas.

Los datos cromosómicos existentes en Leguminosas americanas revelan una gran variación entre géneros, especies de un mismo género y taxones infraespecíficos, encontrando números haploides (n) que varían entre 5 y 15. Goldblatt (1981) analizó los resultados cromosómicos y dedujo que el número básico ancestral en la familia es $\mathrm{x}=7$. Este autor propuso que por procesos de poliploidía se originaron, posteriormente, especies con $2 \mathrm{n}=28 \quad(\mathrm{x}=14)$; asimismo las tribus más especializadas son las que poseen números cromosómicos más bajos, los que habrían surgido por reducción aneuploide.

Durante la presente revisión, en concordancia con Goldblatt (1981), se encontró que aunque en la mayoría de los casos los estudios citológicos sólo indican el número cromosómico, estos datos son sumamente útiles para delimitar grupos dentro de la familia.

Los cambios cromosómicos pueden producirse, durante el proceso evolutivo, en cualquier sentido y muchas veces en forma cíclica. En citogenética, a diferencia de otras disciplinas, no hay leyes o principios establecidos para utilizar los términos ancestral o derivado, a excepción de la poliploidía cuya reversión es poco frecuente (Jackson 1971; Stebbins 1971; Poggio \& Naranjo 2004).

En este trabajo se realiza una revisión de las características cromosómicas representativas descriptas hasta el momento en leguminosas americanas. El análisis de estos datos en conjunto con la caracterización taxonómica y las filogenias moleculares permitirá establecer hipótesis acerca de los cambios cromosómicos ocurridos durante el proceso de divergencia y especiación de la familia.

\section{Materiales y Métodos}

Los números cromosómicos fueron relevados de Goldblatt (1981) y de la base de datos Trópicos, Missouri Botanical Garden, USA (http://mobot.mobot.org/W3T/Search/ ipcn.html); mientras que para el tamaño del genoma se consultó Benett \& Leitch (2003), actualizada en http://www.rbgkew.org.uk/cval/ homepage.html.

La evolución de los números cromosómicos fue estudiada en base a uno de los cladogramas propuestos por Lewis et al. (2005), el cual representa un resumen de los estudios filogenéticos realizados en Leguminosae en base a secuencias nucleares y de cloroplasto. Sobre este cladograma se optimizó el número gamético, que fue tratado como un carácter multiestado no ordenado. Para la optimización se utilizó el programa WinClada (Nixon 2002). 


\section{Resultados y Discusión}

\section{Modelos de evolución cromosómica}

Para explicar la variación encontrada en los números somáticos (2n) y gaméticos (n) se postulan dos modelos de evolución cromosómica, los cuales no son mutuamente excluyentes: paleopoliploidía a partir de números cromosómicos bajos y disploidía (creciente, decreciente).

El modelo de la paleopoliploidía postula que las especies basales, probablemente ya extintas, poseían $2 \mathrm{n}=14$ (número básico $\mathrm{x}=7$ ), siendo poliploides la mayoría de las especies actuales con $2 \mathrm{n}=28(\mathrm{n}=14 ; 4 \mathrm{x}, \mathrm{x}=7)$ y un número básico derivado de $\mathrm{x}=14$. Procesos de hibridación y poliploidía secundarios pueden originar series poliploides $(2 \mathrm{n}=28,56)$ y nuevos números básicos secundarios. De acuerdo a esta propuesta la mayoría de las especies actuales de Leguminosas serían poliploides.

La disploidía se refiere a la existencia de cambios cromosómicos disploides decrecientes o crecientes. En este caso los rearreglos cromosómicos (fusiones o fisiones cromosómicas) disminuyen o aumentan el número de cromosomas y alteran su morfología. Grehilhuber \& Ehrendorfer (1988) presentan varios modelos de disploidía para explicar variaciones en el número cromosómico. Estos procesos se pueden dar en especies ancestrales diploides, con series descendentes (14 a 12 a $10)$, o ascendentes (14 a 16 a 18), o en niveles más elevados de ploidía (28 a 26 a 24 a 22) llevando a la existencia de series poliploides modificadas $(2 \mathrm{n}=14,28,26,24)$. Por otro lado pueden ocurrir procesos cíclicos, como fusión o fisión, seguidos por poliploidía y, en algunos casos, ocurrencia posterior de disploidía. Es interesante destacar que las fusiones y fisiones mantienen el contenido génico y el tamaño del genoma constantes. En ambos casos se producen cambios en el número de grupos de ligamiento que podrían estar relacionados con el surgimiento de barreras de aislamiento reproductivo y procesos de especiación. En el caso de las fusiones se origina un pequeño fragmento con centrómero que puede perderse o, como fuera postulado para algunos grupos de angiospermas, originar cromosomas supernumerarios, frecuentes en algunas especies de distintos géneros de Leguminosas: Leucaena, Astragalus, Mimosa, Cassia, Millettia, Tephrosia, Indigofera, Desmodium, Erythrina, Vicia, Medicago, Trifolium, Crotalaria, Genista (Tropicos: http:// mobot.mobot.org/W3T/Search/ipcn.html).

La gran variación encontrada en los números cromosómicos puede ser explicada por la actuación conjunta de ambos modelos (paleopoliploidía y disploidía): especies ancestrales con $2 n=14$ pueden originar especies con $2 \mathrm{n}=28$ cromosomas, y la posterior ocurrencia de cambios disploides (crecientes o decrecientes) explicarían la presencia de otros números cromosómicos frecuentes $(26$, 24, 22, etc.) (Fig. 1a).

Además de los dos modelos postulados, también es posible interpretar la variación cromosómica como derivada por hibridación y poliploidia secundaria. El cruzamiento de taxones con número cromosómico $2 n=12$ y $2 n=$ 14 originarían híbridos $2 n=13$ que por poliploidía darían especies de $2 \mathrm{n}=26$ cromosomas, por lo tanto estas especies serían de origen híbrido (alopoliploides), siendo $\mathrm{x}=13$ un número básico secundario o derivado (Fig. 1a), pudiendo surgir por un nuevo evento de poliploidía individuos $2 \mathrm{n}=52$ (poliploidía secundaria). $\mathrm{Si}$ sólo se dispone de los datos cromosómicos es difícil discernir si una especie se originó por disploidia decreciente, o por hibridación y poliploidía secundaria.

Algunos géneros presentan números cromosómicos poco frecuentes (Ej. Ononis: $2 \mathrm{n}=30$ ); en este caso se originan números básicos secundarios $(\mathrm{x}=15)$ por hibridación $\mathrm{y}$ poliploidía. Posteriormente podrían ocurrir cambios disploides (decrecientes o crecientes) dando lugar a $2 \mathrm{n}=28(\mathrm{x}=14)$ o $2 \mathrm{n}=32(\mathrm{x}=16)$ (Fig. 1b).

Para discernir entre los modelos propuestos es necesario un conocimiento completo del grupo desde un enfoque multidisciplinario que incluya, además de los análisis cromosómicos, 


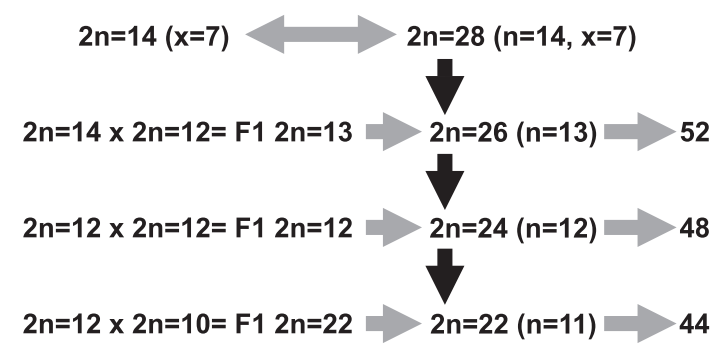

a

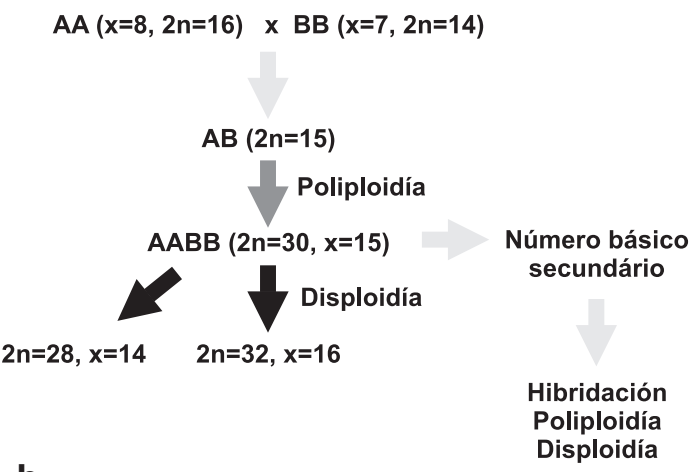

Figura 1 - a. Modelo general de evolución cromosómica en Leguminosae. En gris claro se indican eventos de poliploidía, en negro eventos de disploidía. b. Ejemplo de evolución cromosómica.

estudios morfológicos y moleculares. Solo de esta manera se podrán revelar casos de poliploidía ancestral o críptica, entender las relaciones evolutivas dentro de complejos poliploides y distinguir entre su origen único o recurrente.

Actualmente las técnicas de citogenética molecular, como hibridación in situ, han permitido realizar aportes relevantes en este tipo de estudios (Bennett 1995). Una de las aplicaciones de esta técnica es la obtención de un mapa físico, funcional y estructural del genoma (FISH) utilizando secuencias de distinto origen (Naganowska \& Zielinska, 2002; Sede et al. 2006): Figuras $2 \mathrm{~g}$, h. Mediante esta técnica, en Arachis se realizó el mapeo físico de regiones rRNA aportando evidencias acerca de los progenitores diploides de $A$. hypogaea (Seijo et al. 2004) y, en Vicia, permitió resolver la taxonomía de grupos complejos (Ruijin et al. 2001). Moscone et al. (1999) lograron mediante marcadores moleculares identificar cromosomas en
Phaseolus. Asimismo, con ADN genómico total como sonda (GISH) se pueden revelar homologías específicas, principalmente en secuencias repetidas, posibilitando: reconocer especies parentales en híbridos y poliploides, analizar afinidades genómicas interespecíficas, detectar reestructuraciones cromosómicas y, existencia de apareamiento intergenómico y recombinación (Bennett 1995; Sharma \& Sharma 2001). Antecedente de la aplicación de esta técnica en Leguminosas es el grado de afinidad genómica mostrada entre distintas especies de Phaseolus (Mercado-Ruaro \& Delgado Salinas 2000).

\section{Variación del tamaño del genoma}

El tamaño del genoma es un carácter clave en la biodiversidad, siendo útil en un contexto filogenético y en el análisis de los mecanismos involucrados en la evolución cromosómica (Bennett \& Leitch 2005).

En relación al número de especies que posee la familia Leguminosas, son pocos los datos que incluyen el tamaño del genoma. La búsqueda bibliográfica realizada indicó ca. 600 datos en un rango de 400 a 26,000 Mbp/1C. En la familia el valor $\mathrm{C}$ por genoma básico es en general muy bajo, a excepción de géneros y/o especies especializadas o cultivadas, en las que se encuentra una gran variación intra e interespecífica (http://www.rbgkew.org.uk/ cval/homepage.html; Doyle \& Luckow 2003).

En Papilionoideae hay grupos con muy bajo contenido de ADN, que oscila entre 417$466 \mathrm{Mpb}$ (Vigna, Phaseolus, Lotus). Por otro lado en la tribu Dalbergieae existen representantes con mayor valor C (Dalbergia 1.200 Mbp, Arachis 1.400-3.400 Mbp). Aunque los datos indican la existencia de genomas pequeños en la mayoría de las leguminosas (Doyle \& Luckow 2003), en la tribu Fabeae hay un drástico aumento en el tamaño a nivel diploide y una importante variación interespecífica (2.000-14.000 Mbp, en Lathyrus, Vicia, Lens, Pisum).

Por su parte la subfamilia Mimosoideae todas las especies estudiadas hasta el momento, 
poseen genomas pequeños (Prosopis, Acacia: $200 \mathrm{Mbp}$ a $500 \mathrm{Mbp} / 1 \mathrm{C})$.

En Caesalpinoideae no se conoce el tamaño del genoma del género basal de Leguminosas: Cercis $(2 \mathrm{n}=14)$, pero entre los representantes de la tribu Cercideae, Bauhinia $(2 n=28$ y 26: Fig. $2 f)$ posee un genoma de 588 Mbp. En el resto de las Caesalpinoideae y Mimosoideae estudiadas el valor C oscila entre 600 y 900 Mpb. Sobre esta base Doyle \& Luckow (2003) deducen que las leguminosas basales poseerían genomas pequeños.

\section{Análisis filogenético de la variación cromosómica}

De acuerdo a los antecedentes filogenéticos (Kaas \& Wink 1996; Wojciechowski 2003; Wojciechowski et al. 2004), las subfamilias Papilionoideae y Mimosoideae serian monofiléticas y Caesalpiniodeae seria parafilético (Fig. 3). En el cladograma (Fig. 3) se observa una tendencia hacia la disminución del número cromosómico como resultado de una disploidía decreciente.

Las especies de Caesalpinioideae presentan una gran variación en el número somático, con $2 \mathrm{n}=14,16,20,22,24,26$, y 28 cromosomas. Los géneros con $2 \mathrm{n}=14$ ( $\mathrm{x}=7$ ) como Cassia, Senna y Cercis, estarían indicando que la poliploidía ocurrió temprano en el proceso evolutivo. Cabe destacar que Cercis sería el género de origen más basal en Leguminosas (Goldblatt 1981; Lewis et al. 2005). En Senna y Cassia están presentes ambos niveles de ploidía: $2 \mathrm{n}=14$ y $2 \mathrm{n}=28$ (Fig. $2 \mathrm{~d}$ ), existiendo en este último género especies con $2 n=21$, lo que sugiere la probable presencia de hibridación entre entidades con distintos niveles de ploidía. A su vez en la tribu Detariae, la mayoría de los géneros que crecen en Sudamérica poseen $\mathrm{n}=12$ cromosomas. El análisis de los datos indica que en esta subfamilia habría predominado el proceso de disploidia decreciente ( $\mathrm{n}=14 \mathrm{a} \mathrm{n}=11$ ), existiendo series poliploides en algunos géneros como Caesalpinia $(2 \mathrm{n}=12$ y $2 \mathrm{n}=24)$; asimismo en representantes de Chamaecrista que crecen en Brasil $(2 n=14,16$ y 32$)$, se ha postulado una evolución cromosómica por disploidia y posterior poliploidía (Biondo et al. 2006). En relación a la morfología cromosómica, los estudios realizados en Caesalpinioideae muestran cromosomas pequeños (menor a $3 \mathrm{~m}$ ) y cariotipos simétricos, siendo muy poco frecuente la presencia de cromosomas con centrómero terminal o subterminal (Zanin \& Cangiano 2001).

En la monofilética Mimosoideae, ocurrió un evento principal de evolución del número cromosómico de 14 a 13 (Fig. 3). El género Dinizia presenta $\mathrm{n}=14$, mientras que las tribus Acacieae, Ingaeae y Mimoseae poseen como número gamético más común $\mathrm{n}=13(2 \mathrm{n}=26)$; sobre la base que Dinizia es considerado género basal de la subfamilia (Lewis et al. 2005) el número cromosómico de estas tribus se habría originado por disploidia decreciente a partir de $n=14$. No obstante, es importante mencionar que en Mimoseae hay representantes genéricos con $\mathrm{n}=14$ como Prosopis (Fig. 2a) y Neptunia, y en otros grupos de la subfamilia también varios géneros que presentan series poliploides, como Samanea $(13,26)$, Inga (13, 26) y Acacia $(13,26,39,52,104)$; el recuento $2 n=39$ indicaría la existencia de hibridación entre taxones con distinto nivel de ploidía.

Un caso interesante es Prosopis $(2 \mathrm{n}=28)$, el cual fue considerado un paleopoliploide $(x=7)$; en este género ocurre frecuente hibridación e introgresión interespecífica, y escasa poliploidía (Hunziker et al. 1975; Naranjo et al. 1984; Hunziker et al. 1986). A su vez en representantes de Brasil, Paraguay y Argentina de Mimosa se han registrado distintos niveles de ploidía $(13,26,52)$ (Seijo 2000; Seijo et al. 2004).

Los géneros monotípicos del Sur de Sudamérica: Piptadenopsis y Mimozyganthus presentan $2 \mathrm{n}=28$ cromosomas; ambos son conflictivos desde el punto de vista sistemático y estarían relacionados, de acuerdo a la filogenia molecular propuesta por Luckow et al. (2005), con Prosopidastrum y Leucaena. En este último género hay evidencias citogenéticas, morfológicas y moleculares que 


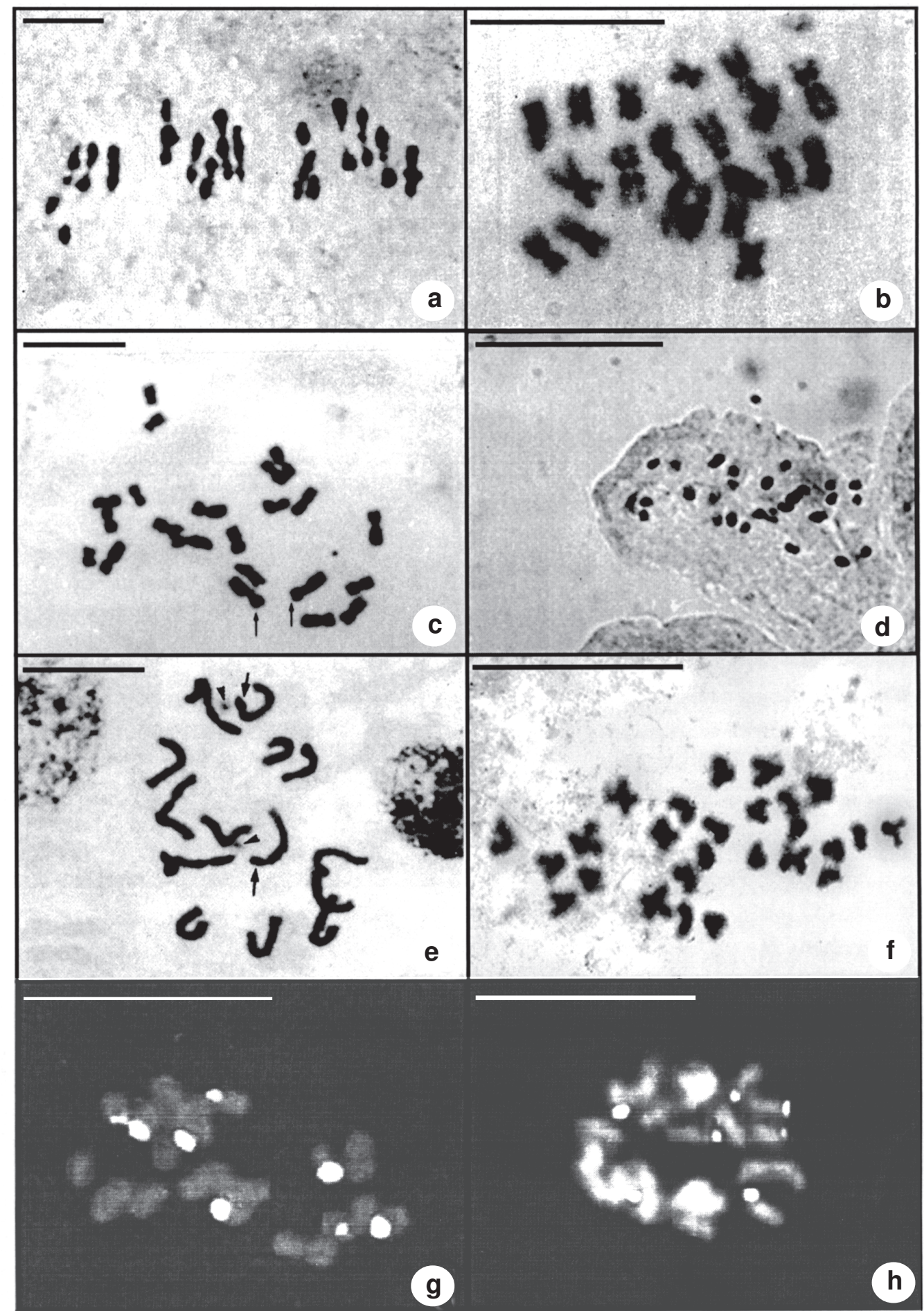

Figura 2 - a. Cromosomas meióticos de Prosopis ruscifolia, 2n=28 (Hunziker et al. 1975); b. bandeo C de células en metafase de Phaseolus coccineus ssp. coccineus, 2n=22 (Castagnaro et al. 1990); c. células somáticas en metafase de Astragalus pehuenches, 2n=22 (Dopchiz et al. 1995); d. metafase de Senna chloroclada 2n=28; e. cromosomas somáticos de Vicia pampicola, 2n=14 (Naranjo et al. 1998); f. metafase de Bauhinia uruguayensis, 2n=26; g. hibridación in situ de Galactia fiebrigiana, 2n=20 (Sede et al. 2006); h. hibridación in situ de Collaea stenophylla, 2n=20, usando como sonda la región rDNA pTa71 (Sede et al. 2006). La barra en las figuras a-f representa $10 \mu \mathrm{m}$ y g-h $5 \mu \mathrm{m}$ 
sugieren que la hibridación interespecífica y poliploidía han sido relevantes en su evolución $(\mathrm{n}=13,26,52,104 / 14,28,56,112)$; como antecedente se indica que Leucaena presenta especies de origen híbrido homoploide y poliploide, y polimorfismo numérico para cromosomas accesorios (cromosomas B), lo cual complica los análisis convencionales de relaciones especificas tanto a nivel taxonómico como evolutivo (Hughes et al. 2002; Boff \& Schifino Wittman 2003). Calliandra es atípico en la tribu Ingeae, por ser el único representante genérico con $2 n=16$ cromosomas.

Papilionoideae constituye un grupo monofilético que habría aparecido hace 45-50 millones de años (Wojciechowski 2003). En esta subfamilia los grupos más derivados presentan reducción del número básico de 14 a 7, pasando por números gaméticos de 11 y 8 (Fig. 3).

A continuación se detallan antecedentes de variación cromosómica en algunos géneros de esta subfamilia.

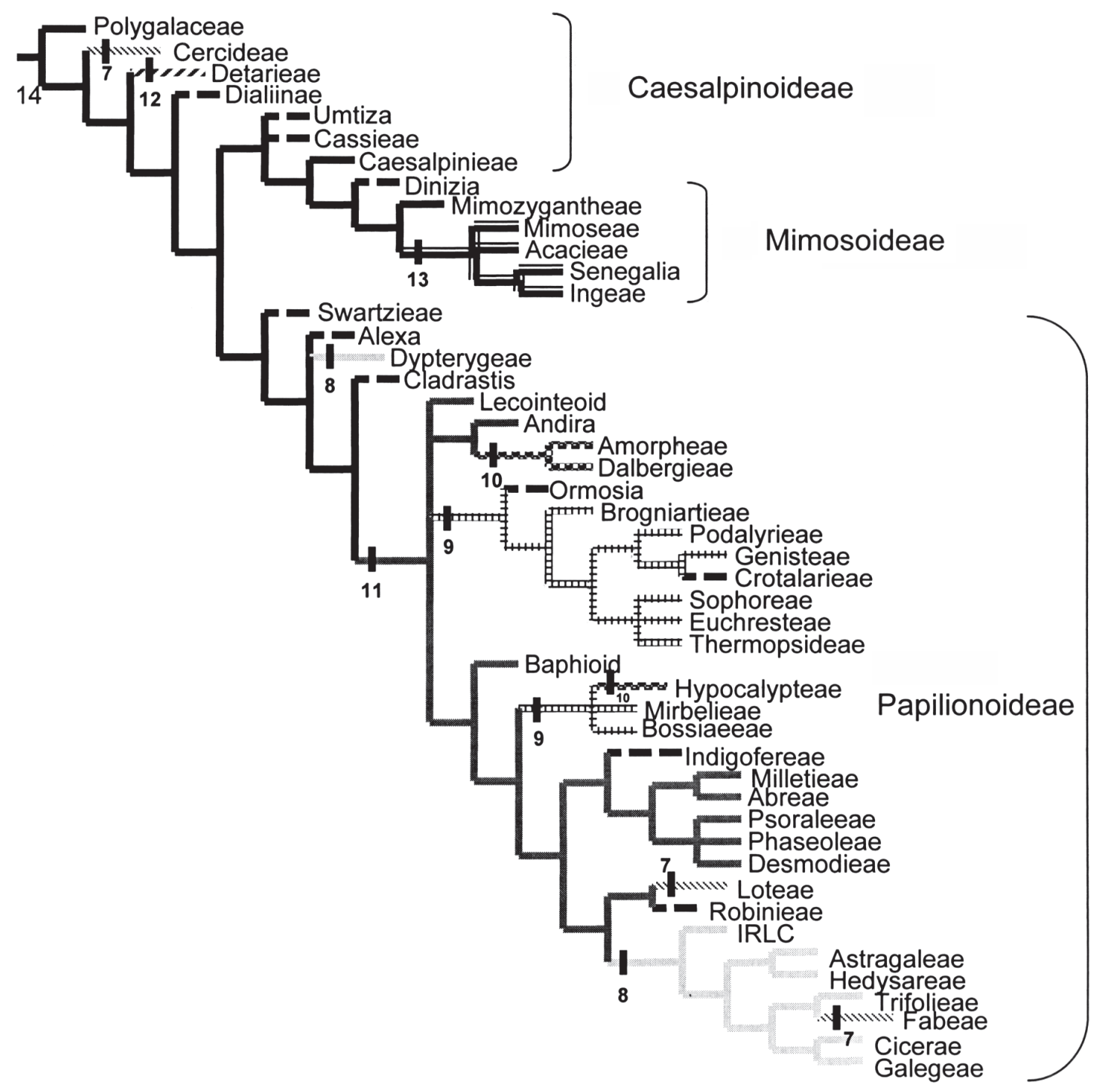

Figura 3 - Cladograma de Leguminosae (Lewis et al. 2005), con el carácter "número cromosómico" optimizado; los estados se indican con distintos colores de ramas, la línea punteada representa una reconstrucción ambigua. 
Lupinus (tribu Genisteae) presenta una gran variación cromosómica y series poliploides $(\mathrm{n}=12,18,19,20,21,24,26,36,38,40,42,48,50)$. En este género existirían varios números básicos, surgidos por cambios disploides que habrían originado series poliploides: $x=6(2 n=12,18,24$, $36,48,96) ; x=5(2 n=40,50,100) ; y x=9(2 n=18$, $36,54,52)$. Algunos números gaméticos (ej. $19,21)$ son números básicos secundarios que surgieron por alopoliploidía. El contenido de ADN en este género oscila entre 0.97 y 2.44 pg (2C) (Naganowska et al. 2003).

En la tribu Crotalariae, Crotalaria presenta como números cromosómicos más frecuentes $2 n=16,32(x=8)$; sin embargo hay algunas especies con $2 n=14(x=7)$. En este género las características cromosómicas corroboran la agrupación de especies en distintas secciones (Almada et al. 2006).

En Phaseoleae, el género Phaseolus presenta generalmente $2 \mathrm{n}=22(\mathrm{x}=11)$, aunque se han registrado especies con $2 n=20$ (Fig. 2b). El tamaño del genoma varía entre 1.5 a 1.98 pg (2C). Esta variación estaría relacionada a cambios en cantidad y posición de heterocromatina y a características ecológicas y climáticas (Castagnaro et al. 1990; Mercado-Ruaro \& Delgado Salinas 1998; Mercado-Ruaro \& Delgado Salinas 2000). Los registros cromosómicos en Camptosema son $2 \mathrm{n}=22 \mathrm{y}$ 2n=20 (Turner \& Irwin 1961; Coleman \& Smith 1969; Sede et al. 2003; Sede et al. en prensa); esta variación numérica se observa principalmente en especies que crecen en el Sur de Sudamérica y, que además poseen caracteres morfológicos relacionados con Galactia y Collaea, dificultando la delimitación genérica. Asimismo, estos dos últimos géneros presentan también registros con $n=10,2 n=20$ (Turner 1956; Krapovickas 1965; Fernández 1977; Coleman \& Demenezes 1980; Seijo \& Vanni 1999; Bossi \& Daviña 2000; Sede et al. 2003). Las especies hasta el momento estudiadas del complejo Galactia-Collaea-Camptosema han mostrado variación interespecífica, y pequeño tamaño de cromosomas, ambos tipos de datos están imposibilitado utilizar la localización de zonas rDNA mediante FISH para la diferenciación genérico-específica (Fig. 2g-h) (Sede et al. 2006).

Astragalus (tribu Astragaleae) presenta una gran variación en el número, tamaño y morfología cromosómica $(n=6,8,10,11,12$, 13, 14,15). Este género posee cariotipos bimodales (Fig. 2c), variación en el tipo y posición de satélites, y en el contenido de ADN (2C $=2.8-3.7 \mathrm{pg})$ (Dopchiz et al. 1995).

El género Arachis (tribu Dalbergieae, sensu Lavin et al. 2001; Klitgaard \& Lavin 2005) posee la mayoría de las especies con $2 n=2 x=20(x=10)$ cromosomas, sin embargo, Lavia (1998) describió un nuevo número básico $(\mathrm{x}=9 ; 2 \mathrm{n}=18)$. Esta autora propone que $\mathrm{x}=9$ seria derivado de $\mathrm{x}=10$ por aneuploidía (disploidia decreciente).

En la tribu Fabeae, Vicia posee gran variación en el número y morfología cromosómica (Fig. 2e): 2n=10, 12, 14, 24, 28 $(\mathrm{x}=5,6,7)$. Su contenido de ADN varía entre 3.66-27.07 pg (2C) (Naranjo et al. 1998); mientras que en Lathyrus el numero cromosómico es constante $(2 \mathrm{n}=14)$ y las especies sudamericanas se diferencian por su formula cariotípica (Seijo \& Fernández 2003). Ambos géneros se caracterizan por poseer el mayor tamaño del genoma descrito para leguminosas.

\section{Conclusiones}

La revisión de los datos cromosómicos existentes en Leguminosas americanas revelan una gran variación en el número gamético ( 5 a 15), morfología cromosómica y tamaño del genoma $(0.4-15 \mathrm{pg} / 1 \mathrm{C})$. La variación encontrada puede explicarse por varios modelos: paleopoliploidia, disploidía (creciente o decreciente), hibridación y poliploidía secundaria o, su combinación. Dado que los cambios cromosómicos pueden ocurrir en forma cíclica, se analizaron los números gaméticos conjuntamente con los datos filogenéticos moleculares con el propósito de validar las hipótesis propuestas, y comprender los mecanismos de evolución cromosómica que han ocurrido en los distintos grupos. 
Los datos indican que los dos modelos propuestos en forma combinada, han sido importantes en la evolución de la familia, existiendo una tendencia a la disminución del número cromosómico en la mayoría de los grupos. El análisis del tamaño del genoma sugiere que las leguminosas basales poseían genomas pequeños, mientras que ha ocurrido un aumento del contenido de ADN en los taxones más derivados (Fabeae).

La evaluación realizada permite establecer la dirección de los cambios cromosómicos (variación numérica y tamaño del genoma) y postular que la hibridación y poliploidia jugaron un rol preponderante en la evolución de la Familia. Los resultados de este estudio en entidades conflictivas de Leguminosae serán de gran utilidad para establecer grupos naturales.

\section{Agradecimilentos}

Se agradece al Comité organizador del IX Congreso Latinoamericano de Botánica (Santo Domingo, República Dominicana), por la invitación recibida para presentar este trabajo en el marco del Simposio de Leguminosas. Asimismo, LP hace extensivo su agradecimiento a la Agencia Nacional de Promoción Científica y Tecnológica (PICT 14119), al Consejo Nacional de Investigaciones Científicas y Técnicas (CONICET: PIP 5927) y a la Facultad de Ciencias Exactas y Naturales, UBA, y RHF a CONICET (PIP 5560) y a Myndel Botanica Foundation, Convocatoria 2002, 2004 y 2005, por los subsidios otorgados para recolectar, procesar $\mathrm{y}$ analizar las muestras del material que ha sido base de esta presentación. Los autores también quieren expresar su gratitud al Sr. Diego Fink por la configuración realizada de la lámina de fotos de cromosomas y FISH.

\section{REFERENCIAS BIBLIOGRÁFICAS}

Almada, R. D.; Daviña, J. R. \& Seijo, J. G. 2006. Karyotype analysis and chromosome evolution in southernmost South American species of Crotalaria (Leguminosae). Botanical Journal of the Linnean Society 150: 329-341.
Benett, M. D. \& Leitch, I. J. 2003. Plant DNA C-values database. Royal Botanic Gardens, Kew. www. rbgkew.org/cval/ homepage.html

Bennett, M. D. 1995. The development and use of genomic in situ hibridization (GISH) as a new tool in plant biosystematics. In: Brandham, P. E. \& Bennett, M. D. Kew Chromosome Conference IV. Royal Botanic Gardens, Kew. Pp. 167-183.

Bennett, M. D. \& Leitch, I. J. 2005. Genome size evolution in plants. In: Ryan Gregory, T. The evolution of genome. Elsevier Academic Press: 740.

Biondo, E.; Miotto, S. T. S. \& SchifinoWittmann, M. T. 2006. Cytogenetics of species of Chamaecrista (LeguminosaeCaesalpiniodeae) native from southern Brazil. Botanical Journal of the Linnean Society 150: 429-439.

Boff T. \& Schifino-Wittman, M. T. 2003. Segmental allopolyploidy and paleopolyploidy in species of Leucaena Benth: evidence from meiotic behavior analysis. Hereditas 138(1): 27-35.

Bossi, F. S. \& Daviña, J. R. 2000. Cromosomas de cuatro especies de Galactia. Bonplandia 20: 175-179.

Castagnaro, A., Poggio, L. \& Naranjo, C. A. 1990. Nuclear DNA content variation in Phaseolus (Fabaceae). Darwiniana 30: 195-200.

Coleman, J. R. \& Demenezes, E. M. 1980. Chromosome numbers in Leguminosae from the State of Sao Paulo, Brazil. Rhodora 82: 475-481.

Coleman, J. R. \& Smith, L. B. 1969. Chromosome numbers of some Brazilian angiosperms. Rhodora 71: 548-551.

Dopchiz, L.; Gomez Sosa, E. \& Poggio, L. 1995. Karyotype and nuclear DNA content of six species of Astragalus. Cytologia 60: 329-335.

Doyle, J. J. \& Luckow, M. A. 2003. The rest of the iceberg. Legume diversity and evolution in a phylogenetic context. Plant Physiology 131: 900-910. 
Fernández, A. 1977. Números cromosómicos en Angiospermas. Hickenia 1: 83-86.

Goldblatt, P. 1981. Cytology and the phylogeny of Leguminosae. In: Polhill, R. M. \& Raven, P. H. Advances in legume systematics. Royal Botanic Gardens, Kew, 2: 427-463.

Grant, V. 1985. The evolutionary process. W.H. Freeeman and Co., San Francisco. 499p.

Grehilhuber, J. \& Ehrendorfer, F. 1988. Karyological approaches to plant taxonomy. ISI Atlas of Science. Vol. 1.

Hughes C. E.; Bailey, C. D. \& Harris, S. A. 2002. Divergent and reticulate species relationships in Leucaena (Fabaceae) inferred from multiple data sources: insights into polyploid origins and nrDNA polymorphism. American Journal of Botany 89: 1057-1073.

Hunziker, J. H.; Poggio, L.; Naranjo, C. A.; Palacios, R. A. \& Andrada, B. 1975. Cytogenetics of some species and natural hybrids in Prosopis (Leguminosae). Canadian Journal of Genetics and Cytology 17: 253-262.

Hunziker, J. H.; Saidman, B. O.; Naranjo, C. A.; Palacios, R. A.; Poggio, L. \& Burghardt, A. D. 1986. Hybridization and genetic variation of Argentine species of Prosopis. Forest Ecol. Management 16: 301-315.

Jackson, R. C. 1971. The karyotype in systematics. Annual Review of Ecology and Systematic 2: 327-368.

Kaas, E. \& Wink, M. 1996. Molecular evolution of the Leguminosae: phylogeny of the three subfamilies based on rbc-L sequences. Biochemical Systematics and Ecology 24: 365-378.

Klitgaard, B. B. \& Lavin, M. 2005. Tribe Dalbergieae sens. lat. In: Lewis, G. P.; Schrire, B. D.; Mackinder, B. \& Lock, M. Legumes of the world. Royal Botanic Gardens, Kew. Pp. 307-335.

Krapovickas, A. 1965. Recuentos cromosómicos de leguminosae. Kurtziana 2: 91-94.

Lavia, G. I. 1998. Karyotype of Arachis palustris and $A$. praecox (Section Arachis). Two species with basic number $\mathrm{x}=9$. Cytologia 63: 177-181.
Lavin, M.; Pennington, R.; Klitgaard, B. B.; Sprent, J.; De Lima, H. \& Gasson, P. 2001. The dalbergioid legumes (Fabaceae): delimitation of a pantropical monophyletic clade. American Journal of Botany 88: 503-533.

Leitch, I. J.; Chase, M. W. \& Benett, M. D. 1998. Phylogenetic analysis of DNA Cvalues provides evidence for a small ancestral genome size in flowering plants. Annals of Botany 82: 85-94.

Lewis, G. P.; Schrire, B. D.; Mackinder, B. \& Lock, M. (eds). 2005. Legumes of the world. Kew: 577 p.

Luckow, M.; Fortunato, R.; Sede, S. \& Livshultz, T. 2005. The phylogenetic affinities of two mysterious monotypic mimosoids from Southern South America. Systematic Botany 30: 585-602.

Mercado-Ruaro, P. \& Delgado Salinas, A. 1998. Karyotypic studies on species of Phaseolus (Fabaceae: Phaseolinae). American Journal of Botany 85: 1-9.

2000. Cytogenetic studies in Phaseolus

L. (Fabaceae). Genetics and Molecular Biology 23(4): 985-987.

Moscone, E. A.; Klein, F.; Lambrou, M.; Fuchs, J. \& Schweizer, D. 1999. Quantitative karyotyping and dual color FISH mapping of $5 \mathrm{~S}$ and $18 \mathrm{~S} 25 \mathrm{~S}$ rDNA probes in the cultivated Phaseolus species (Leguminosae). Genome 42: 1224-1233.

Naganowska, B.; Wolko, B.; Liwska, E. \& Kaczmarek, Z. 2003. Nuclear DNA content variation and species relationships in the genus Lupinus (Fabaceae). Annals of Botany 92: 349-355.

Naganowska, B. \& Zielinska, A. 2002. Physical mapping of 18S-25S rDNA and $5 \mathrm{~S}$ rDNA in Lupinus via fluorescent in situ hybridization. Cellular and Molecular Biology Letters 7: 665-670.

Naranjo, C. A.; Ferrari, M.; Palermo, A. \& Poggio, L. 1998. Karyotype DNA content and meiotic behavior in five South American species of Vicia (Fabaceae). Annals of Botany 82: 757-764. 
Naranjo, C. A.; Poggio, L. \& Enus Zeiger, S. 1984. Chromatography of phenols, morphology and cytogenetics in three species and natural hybrids of Prosopis (P.affinis, P.alba and P. nigra) (Leguminosae, Mimosoidae). Plant Systematics and Evolution 144: 257-276.

Nixon, K. C. 2002. WinClada, 1.00.08. Ithaca, NY.

Poggio, L. \& Naranjo, C. A. 2004 Citogenética. In: Echenique, V.; Rubinstein, C. \& Mroginski, L. Biotecnología y mejoramiento vegetal. Editorial INTA. Pp. 69-79.

Ruijin, L.; Taylor, S. \& Jenkins, G. 2001. Unravelling the phylogeny of tetraploid Vicia amoena (Fabaceae) and its diploid relatives using chromosomal landmarks. Hereditas 134: 219.

Sede, S.; Fortunato, R. \& Poggio, L. 2006. Chromosome evaluation of southern South American species of Camptosema and allied genera (Diocleinae-PapilionoideaeFabaceae). Botanical Journal of the Linnean Society 152: 235-243.

Sede, S.; Greizerstein, E. J.; Dezi, R.; Fortunato, R. \& Poggio, L. 2003. Chromosome studies in the Complex Galactia-Collaea-Camptosema (Fabaceae). Caryologia 56: 295-301.

Seijo, G. 2000. Números cromosómicos de especies de Mimosa (Leguminosae) de Paraguay. Bonplandia 10: 163-167.

Seijo, G. \& Vanni, R. 1999. Números cromosómicos en Leguminosas de Paraguay. Boletin de la Sociedad Argentina de Botanica 34: 119-122.

Seijo, J. G. \& Fernández, A. 2003. Karyotype analysis and chromosome evolution in South American species of Lathyrus (Leguminosae). American Journal of Botany 90: 980-987.
Seijo, J. G.;Lavia, G.I.;Fernández, A.; Krapovickas, A.; Ducasse, D. \& Moscone, E. A. 2004. Physical mapping of the $5 \mathrm{~S}$ and $18 \mathrm{~S}-25 \mathrm{~S}$ rRNA genes by FISH as evidence that Arachis duranensis and A. ipaensis are the wild diploid progenitors of $A$. hypogaea (Leguminosae). American Journal of Botany 91: 1294-1303.

Sharma, A. K. \& Sharma, A. 2001. Chromosome painting. Principles, strategies and scope. Kluwer Academic Publishers, 179p.

Soltis, D. E.; Soltis, P. S.; Benett, M. D. \& Leitch, I. J. 2003. Evolution of genome size en the angiosperms. American Journal of Botany 90: 1596-1603.

Stebbins, G. L. 1971. Chromosomal evolution in higher plants. Addison Wesley Publ., Reading, Massachusetts, 216p.

Turner, B. L. 1956. Chromosome numbers in the Leguminosae I. American Journal of Botany 43: 577-581.

Turner, B. L. \& Irwin, H. S. 1961. Chromosome numbers of some brazilian Leguminosae. Rhodora 63: 16-19.

Wojciechowski, M. F. 2003. Reconstructing the phylogeny of legumes (Leguminosae): an early $21^{\text {st }}$ century perspective. In: Klitgaard, B. B. \& Bruneau, A. Advances in legume systematics, part 10. Royal Botanic Gardens, Kew. Pp. 5-35.

Wojciechowski, M. F.; Lavin, M. \& Sanderson, M. 2004. A phylogeny of legumes (Leguminosae) based on analysis of the plastid matK gene resolves many wellsupported subclades within the family. American Journal of Botany 91: 1846-1862.

Zanin, L. A. \& Cangiano, M. A. 2001. El cariotipo de Hofmannseggia glauca (Fabaceae). Darwiniana 39: 11-13. 